\title{
Malignant mesothelioma clinical trial combines immunotherapy drugs
}

\author{
Monica S Chatwal ${ }^{1}$ \& Tawee Tanvetyanon*,2 \\ ${ }^{1}$ Division of Medicine, Division of Hematology/Oncology, University of South Florida/ H Lee Moffitt Cancer Center, Tampa, FL \\ 33612, USA \\ ${ }^{2}$ Thoracic Oncology Department, H. Lee Moffitt Cancer Center, FL 33612, USA \\ * Author for correspondence: Tel.: +813 745 3050; Fax: +813 745 3027; tanvett@moffitt.org
}

"while the full published results from MAPS2 and NIBIT-Meso1 studies are still pending, the use of combined checkpoint inhibitors appears promising for mesothelioma. The approach opens many potential therapeutic avenues for advanced mesothelioma. "”

Immunotherapy by checkpoint inhibitor is effective for a number of solid tumors including malignant mesothelioma. Studies utilizing single-agent PD-1 or PD-L1 inhibitor for mesothelioma have reported tumor response rates in approximately $10-20 \%$ of patients treated. Given the success of combining these agents with CTLA-4 inhibitor in melanoma, there is a strong rationale to study it in mesothelioma. Recently results from clinical trials investigating this approach have been released. Though limited by small sample size, the studies conclusively demonstrated feasibility and suggested a modestly higher tumor response rate than one would expect from treatment with single-agent PD-1 or PD-L1 inhibitor. Nevertheless, toxicity was also increased. Immunotherapy-related deaths due to encephalitis, renal failure and hepatitis were observed. Further studies are warranted.

First draft submitted: 30 November 2017; Accepted for publication: 9 January 2018; Published online: 23 February 2018

Keywords: immune checkpoint inhibitor $\bullet$ immunotherapy $\bullet$ ipilimumab • mesothelioma $\bullet$ nivolumab

Malignant mesothelioma is an uncommon thoracic malignancy diagnosed in approximately 3000 cases each year in the USA [1]. Typically associated with asbestos exposure, mesothelioma disproportionately affects older patients, who often have significant comorbidity and advanced stage of the disease, making treatment challenging. Improvement in systemic therapy, the mainstay of treatment, has been slow, with pemetrexed being the last agent to gain regulatory approval by the US FDA, in 2004 [2]. Today, the first-line systemic therapy remains pemetrexed-based chemotherapy. Typical median overall survival with this treatment as reported from clinical trials using pemetrexed cisplatin with or without bevacizumab typically ranges from 1 to 1.5 years [3]. For patients with recurrent or refractory disease, subsequent therapeutic options are limited. There is a clear need for additional treatment options beyond chemotherapy.

During the past decade, there has been a rapid advance in anti-cancer immunotherapy, specifically through blockade of the immune checkpoints, PD-1 or PD-L1 and CTLA-4 [4]. Immune checkpoint inhibitors have already gained regulatory approval for treatment of many cancers. For mesothelioma, there has been much enthusiasm for these agents, given the inflammatory phenotype and reported 20 to 40\% PD-L1 expression of the mesothelioma and stromal cells [5]. To date, several studies have documented the efficacy of single-agent immunotherapy in mesothelioma. For example, pembrolizumab, an anti-PD-1, produced a response rate of approximately $20 \%[6,7]$. Nivolumab, another anti-PD-1 agent, produced a response rate of $15 \%$ in a small cohort of pretreated patients [8]. Avelumab, an anti-PD-L1 with antibody-dependent cell mediated cytotoxicity, yielded $9.4 \%$ partial response rate [9]. Given that combining anti-PD-1 or anti-PD-L1 therapy with CTLA-4 agent may produce synergism, thus further enhancing the efficacy of treatment, there has been an interest investigating the use of combined checkpoint inhibitors as a treatment for mesothelioma. 


\begin{tabular}{|c|c|c|}
\hline Characteristics & Single-agent immunotherapy & Combined immunotherapy \\
\hline \multicolumn{3}{|l|}{ MAPS2 } \\
\hline Treatment schedule & $3 \mathrm{mg} / \mathrm{kg}$ every 2 weeks & $3 \mathrm{mg} / \mathrm{kg}$ every 2 weeks and $1 \mathrm{mg} / \mathrm{kg}$ every 6 weeks \\
\hline $\mathrm{N}$ & 63 & 62 \\
\hline Objective response rate (\%) & 17.5 & 24.2 \\
\hline Grade 5 toxicity (\%) & 0 & 4.8 \\
\hline \multicolumn{3}{|l|}{ NIBIT-Meso1 } \\
\hline Agent & N/A & Durvalumab and tremelimumab \\
\hline Treatment schedule & $\mathrm{N} / \mathrm{A}$ & $\begin{array}{l}20 \mathrm{mg} / \mathrm{kg} \text { every } 4 \text { weeks for } 13 \text { doses and } 1 \mathrm{mg} / \mathrm{kg} \text { every } \\
4 \text { weeks for } 4 \text { doses }\end{array}$ \\
\hline
\end{tabular}

N/A: Not applicable.

Recently, partial results from two clinical trials investigating combined checkpoint inhibitors have been presented at international meeting (Table 1). The first clinical trial is the MAPS2 study which evaluated treatment with singleagent nivolumab or nivolumab plus ipilimumab (anti-CTLA-4) in previously treated mesothelioma [10]. In this study, patients with pleural mesothelioma, regardless of PD-L1 expression, were randomized to receive nivolumab $3 \mathrm{mg} / \mathrm{kg}$ once every 2 weeks with or without ipilimumab $1 \mathrm{mg} / \mathrm{kg}$ every 6 weeks until disease progression or unacceptable toxicity. The results showed that tumor response rate was numerically higher among patients assigned to receive combined immunotherapy. Among patients receiving single-agent nivolumab, the response rate was $17.5 \%$ (95\% CI: 8.1-26.8). Whereas, among those receiving nivolumab plus ipilimumab, the response rate was $24.2 \%$ (95\% CI: $13.5-34.9)$. Nevertheless, serious adverse events were also more frequent in the combined immunotherapy arm. Grade $\geq 3$ toxicities were reported in $9.5 \%$ of patients in the single-agent nivolumab arm, compared with $21.3 \%$ in the combined arm. The most notable increase in side effects was diarrhea, reported in 19.7 versus $6.3 \%$ and pruritus, reported in 11.5 versus $1.6 \%$, respectively. Furthermore, there were three treatmentrelated deaths reported in the combined arm due to fulminant hepatitis, metabolic encephalopathy, and renal failure each, while there was no treatment-related death reported in the single agent arm.

The second clinical trial is the NIBIT-Meso1 study [11]. The NIBIT-Mesol is a single-arm study evaluating the use of durvalumab (anti-PD-L1) plus tremelimumab either as first- or second-line treatment for pleural or peritoneal mesothelioma. Regardless of PD-L1 status, 40 patients with a median age of 64 years were treated. Overall response rate was $25 \%$. Grade 3 or 4 toxicity occurred in $17.5 \%$ of patients. Although the study does not contain a single-agent durvalumab arm, this result suggests a somewhat higher tumor response rate than one would expect from single-agent anti-PD-L1.

The investigators in the MAPS2 and NIBIT-Meso1 studies are to be congratulated for their significant work enrolling patients with this uncommon malignancy. Although the final reports of both studies are still not available, taken together, data convincingly demonstrate the efficacy of combined checkpoint inhibitors for mesothelioma, yielding an objective response rate of approximately $25 \%$. This number appears higher than the response rate reported historically from single-agent PD-1 or PD-L1 inhibitor treatment, which ranges from 10-20\%. However, the toxicity from combined immunotherapy in mesothelioma is clearly elevated, approximately doubling the incidences of grade $\geq 3$ toxicities. Most notable is the treatment-related death associated with ipilimumab plus nivolumab due to immune-mediated complications such as encephalitis.

To put these results into perspective, it will help to consider the results from a Phase III study of ipilimumab plus nivolumab as compared with nivolumab alone for melanoma [12]. That pivotal study reported $58 \%$ objective response rate with the combined immunotherapy, compared with $44 \%$ with single-agent nivolumab. Although the incidence of grade $\geq 3$ toxicity was higher, 59 versus $21 \%$, there were only 2 treatment-related deaths out of the 313 patients $(0.6 \%)$ treated. Although the incremental gains of efficacy as well as grade $\geq 3$ toxicity from combined 
immunotherapy for mesothelioma appears somewhat comparable to those observed for melanoma, the incidence of treatment-related death seems much higher than one would have expected. Nevertheless, due to the small sample size, additional studies will be needed to understand if the observed treatment-related death is a valid concern.

Currently, it can be stated that pembrolizumab is approved as a treatment for mesothelioma in the subsequentline setting, providing that mismatch repair (MMR) protein deficiency or high microsatellite instability (MSI) is identified [13]. MMR deficient or MSI high tumors are more responsive to PD-1 blockades than MMR proficient tumors because they tend to harbor more somatic mutations and therefore more neoantigens. Unfortunately, in the case of mesothelioma patients, testing for MMR or MSI has not been routinely performed and the reported prevalence of high MSI is low, estimated only about 3\% [14]. Therefore for most mesothelioma patients, there remains no established role of checkpoint immunotherapy at this time. Nevertheless, with an improvement in predictive biomarker identification, it is likely that single-agent immunotherapy will soon find its role to benefit more mesothelioma patients. However, for combined immunotherapy, its role remains to be better defined.

One immediate area of interest is the use of biomarker status to select patients who will benefit more from combined immunotherapy than from single-agent immunotherapy. This population may be those who are unlikely to respond to single-agent immunotherapy such as those with low PD-L1 expression. The analysis integrating PDL1 status from the MAPS study will provide valuable insight to this potential. The other area of interest is toxicity. It is important to note that in diseases such as small cell or thymic carcinoma, immunotherapy uniquely results in serious autoimmune complications [15,16]. Additional experiences of combined immunotherapy in mesothelioma will be needed to confirm its safety. As with any treatment, the justification for the treatment will depend on several factors, not only efficacy and toxicity but also the availability of alternative therapeutic options. Finally, the question still remains about timing of immunotherapy. Many clinical trials are ongoing. For instance, a clinical trial comparing chemotherapy versus ipilimumab plus nivolumab (NCT02899299) is being conducted as firstline treatment setting. In this setting, treatment will need to deliver response quickly and reliably especially for patients with rapidly progressive disease. Other studies using combined chemotherapy such as antimesothelin-based vaccine CRS207 plus pembrolizumab (NCT03175172) as second- or third-line therapy for nonsarcomatoid pleural mesothelioma is ongoing and patients are encouraged to participate in these trials whenever possible in order to facilitate advance in the treatment of this uncommon disease.

In summary, while the full published results from MAPS2 and NIBIT-Meso1 studies are still pending, the use of combined checkpoint inhibitors appears promising for mesothelioma. The approach opens many potential therapeutic avenues for advanced mesothelioma. For a lethal disease without standard treatment options beyond initial chemotherapy, the use of the combined immunotherapy offers hope. However, additional studies will be needed.

Financial \& competing interests disclosure

The authors have no relevant affiliations or financial involvement with any organization or entity with a financial interest in or financial conflict with the subject matter or materials discussed in the manuscript. This includes employment, consultancies, honoraria, stock ownership or options, expert testimony, grants or patents received or pending, or royalties.

No writing assistance was utilized in the production of this manuscript.

\section{References}

1 Beebe-Dimmer JL, Fryzek JP, Yee CL et al. Mesothelioma in the United States: a surveillance, epidemiology, and end results (SEER)-medicare investigation of treatment patterns and overall survival. Clin. Epidemiol. 8, 743-750 (2016).

2 Vogelzang NJ, Rusthoven JJ, Symanowski J et al. Phase III study of pemetrexed in combination with cisplatin versus cisplatin alone in patients with malignant pleural mesothelioma. J. Clin. Oncol. 21(14), 2636-44 (2003).

3 Zalcman G, Mazieres J, Margery J et al. Bevacizumab for newly diagnosed pleural mesothelioma in the Mesothelioma Avastin Cisplatin Pemetrexed Study (MAPS): a randomised, controlled, open-label, Phase III trial. Lancet 387(10026), 1405-1414 (2016).

4 Berger KN, Pu JJ. PD-1 pathway and its clinical application: a 20 year journey after discovery of the complete human PD-1 gene. Gene 638, 20-25 (2018).

5 Thapa B, Salcedo A, Lin X et al. The immune microenvironment, genome-wide copy number aberrations, and survival in mesothelioma. J. Thorac. Oncol. 12(5), 850-859 (2017).

6 Alley EW, Lopez J, Santoro A et al. Clinical safety and activity of pembrolizumab in patients with malignant pleural mesothelioma (KEYNOTE-028): preliminary results from a non-randomised, open-label, Phase 1b trial. Lancet Oncol. 18(5), 623-630 (2017). 
7 Kindler H, Karrison T, Carol Tan Y et al. Phase II trial of pembrolizumab in patients with malignant mesothelioma: interim analysis. J. Thorac. Oncol. 12(S1), S293-S294 (2017).

8 Quispel-Janssen J, Zago G, Schouten R et al. A Phase II study of nivolumab in malignant pleural mesothelioma (NivoMes): with translational research biopies. J. Thorac. Oncol. 12(S1), S292-S293 (2017).

9 Hassan R, Thomas A, Patel MR et al. Avelumab (MSB0010718C; anti-PD-L1) in patients with advanced unresectable mesothelioma from the JAVELIN solid tumor Phase Ib trial: safety, clinical activity, and PD-L1 expression. J. Clin. Oncol. 34(S15), Abstract 8503 (2016).

10 Scherpereel A, Mazieres J, Greillier L et al. Second-or third-line nivolumab versus nivo plus ipilimumab in malignant pleural mesothelioma (MPM) patients: results of the IFCT-1501 MAPS2 randomized Phase II trial. J. Clin. Oncol. 35(S18), Abstract 8507 (2017).

11 Calabro AM, Giannarelli D, Amato G et al. Tremelimumab plus durvalumab in first-or second-line mesothelioma patients: final analysis of the NIBIT-MESO-1 study. J. Thorac. Oncol. 12(S11), S1883 (2017).

12 Wolchok JD, Chiarion-Sileni V, Gonzalez R et al. Overall survival with combined nivolumab and lpilimumab in advanced melanoma. N. Engl. J. Med. 377(14), 1345-1356 (2017).

13 Le DT, Uram JN, Wang H et al. PD-1 blockade in tumors with mismatch-repair deficiency. N. Engl. J. Med. 372(26), 2509-2520 (2015).

14 Bonneville R, Krook MA, Kautto EA et al. Landscape of microsatellite instability across 39 cancer types. Precis. Oncol. 1, 1-15 (2017).

15 Antonia S, Goldberg SB, Balmanoukian A et al. Safety and antitumour activity of durvalumab plus tremelimumab in non-small cell lung cancer: a multicentre, Phase Ib study. Lancet Oncol. 17(3), 299-308 (2016).

16 Giaccone G, Thompson J, Crawford J, McGuire C, Manning M, Subramaniam DS. A Phase II study of pembrolizumab in patients with recurrent thymic carcinoma. J. Clin. Oncol. 34(S15), Abstract 8517 (2016). 